(MDHAQ) 60-symptom checklist may recognize most adverse events of medications.

Objectives: To document recognition of adverse events using a remote weekly electronic MDHAQ completed by patients at home, as a costeffective approach to reduce their morbidity, costs, and mortality

Methods: The MDHAQ includes 0-10 scales for physical function, pain, and patient global assessment, compiled into a 0-30 RAPID3, fatigue and painful joint count, to assess treatment efficacy. The MDHAQ also includes a 60-symptom checklist to recognize comorbidities, review of systems, and symptom reduction; this checklist also recognizes adverse events associated with medications. Weekly remote completion of an electronic MDHAQ at home can monitor both efficacy and adverse events.

Results: A flowsheet from a patient with pulmonary fibrosis monitored over 2018 illustrates remote electronic MDHAQ use. At a first visit of 19 Jan, a routine clinic-based MDHAQ indicated RAPID3 of 14/30, fatigue 6/ 10 , and 10 symptoms (Flowsheet). Treatment with low-dose methotrexate and prednisone led to clinical improvement from 19 Jan to 2 Aug, RAPID3 to 3.5, fatigue 2, and 6 symptoms. On 15 Aug, a pulmonologist discontinued prednisone and methotrexate and prescribed pirfenidone, an anti-fibrosis agent. A telephone call from the patient on 24 Sep indicated distress. A home-completed remote MDHAQ indicated RAPID3 19.5, fatigue 9, 15 symptoms - 7 not reported on 2 Aug were among 16 adverse events listed for pirfenidone. Discontinuation of pirfenidone and resumption of prednisone and methotrexate led to improvement of RAPID3 to 4.2 , fatigue to 1.0 , and resolution of pirfenidone-specific symptoms by 24 Dec, documented with weekly remote electronic MDHAQ (Flowsheet). A brief retrial of pirfenidone indicated an increase of RAPID3 to 6.0 , which was discontinued in a timely manner.

78-year-old man monitored over 2018-all data from self-report on MDHAQ - pirfenidone highlighted (many entries deleted for space considerations) Conclusion: Weekly remote electronic MHDAQ monitoring of a high-risk medication for treatment response and adverse events may provide a cost-effective approach to reduce the morbidity and mortality of adverse events, involving about 10 minutes/weekly or 2 hours of patient time over 12 weeks.

Disclosure of Interests: None declared

DOI: 10.1136/annrheumdis-2019-eular.7698

\section{AB1226 ONE YEAR'S RESULTS OF A PROTOCOLIZED HIGH RESOLUTION RHEUMATOLOGY CLINIC}

Orlando Pompei Fernández ${ }^{1}$, Susana Gil Barato ${ }^{2}$, Belén Álvarez Rodríguez ${ }^{2}$, Juan Ramón De Dios ${ }^{2}$, Margarida Vasques Rocha ${ }^{2}$, Claudia Stoye ${ }^{2}$, Jaime Calvo ${ }^{2}$.

${ }^{1}$ Araba University Hospital, Rheumatology, Vitoria-Gasteiz, Spain; ${ }^{1}$ Araba University Hospital, Rheumatology, Vitoria-Gasteiz, Spain

Background: Mechanical musculoskeletal pathologies put high demands on the Public Health Service, as they affect a significant percentage of the population, and cause both temporary and permanent disabilities.

The specialised High Resolution Rheumatology Clinic (CAR in Spanish) at our centre started up in October 2017, and focuses on the diagnosis and treatment of pathologies which are fundamentally of a mechanical ethology. The centre covers a population of approximately 328,868 inhabitants (Álava 2018 census)

The aim of our study, carried out at the unit over one year, is to determine the patients' epidemiological features.

Objectives: - -To provide the Primary Care Centres with a "fast-track" and immediate care system in order to guide, diagnose, and treat patients who have worsening acute or chronic mechanical afflictions to the musculoskeletal system.

- Diagnosis confirmation, request for non-accessible tests, adaptation of treatments mainly for the primary care doctor.

- To speed up and reduce waiting lists, for both inflammatory rheumatic pathologies and mechanical rheumatic pathologies, by establishing new referral guidelines.

- To draw up protocols in agreement with primary care doctors and related specialists.

- To act as a support for other medical services.

Methods: The main services available are those offered mainly to primary care level. There are 3 clinics a week, attending to approximately 30 new patients per week. 1330 patients are included in the study, seen at the High Resolution Rheumatology Clinic (CAR) over 12 consecutive months (November 2017 - 2018), with non-complex regional pathologies and/or soft tissue rheumatism, which are able to be resolved with one or two visits.
Results: 1330 patients were seen, with an average age of $58 \pm 15.6$ years, $64.64 \%$ female and $35.36 \%$ male, mainly for musculoskeletal ailments. In order of frequency, the visits were for shoulder $(25.79 \%)$, hip $(16.10 \%)$, axial skeleton (15.13\%), ankle/foot (13.06\%), wrist/hand $(12.08 \%)$, knee $(11.21 \%)$, and elbow $(6.63 \%)$.

$61.95 \%$ of the patients were discharged after the first visit, and one year later, this figure rose to $90.15 \%$ of all the patients discharged; only $2.78 \%$ made a return visit after being discharges. $22.26 \%$ were referred to other services, mainly Traumatology and Orthopaedics (10.53\%); Rehabilitation and Physiotherapy (8.72\%); and the Pain Management Unit $(1.65 \%)$. The $3.68 \%$ were referred to the usual Rheumatology department. For $52.33 \%$ of the patients seen, there was no need to request more than one diagnostic tests, even though $12.41 \%$ visited for reasons other than the main one. Moreover, $63.08 \%$ received some kind of infiltration injection. The negative point was that $7.89 \%$ of the referrals had been made from Primary Care to several specialists at the same time; and $7.98 \%$ were consultations regarding traumatic injuries.

Conclusion: Systematising a clinic for mechanical musculoskeletal pathologies which have a high chance of being resolved in the short term, means that the quality of the care given, the waiting times, and the demand on the health service can be improved. Developing these can have important repercussions on waiting lists for other related services, and even for the Rheumatology service itself, allowing more serious cases to be seen earlier. Creating multi-disciplinary units should be encouraged, in order to improve care quality and prevent the various medical services involved from all carrying out fragmented courses of action. New guidelines could be considered, to optimise the care and management of new resources and/or links to other services, as the most prevalent pathologies can be identified.

Most of the patients seen represent the economically active part of the population; there are therefore, repercussions as far as sick leave, disability leave, etc. is concerned.

Disclosure of Interests: Orlando Pompei Fernández: None declared, Susana Gil Barato: None declared, Belén Álvarez Rodríguez: None declared, Juan Ramón De Dios: None declared, Margarida Vasques Rocha: None declared, Claudia Stoye: None declared, Jaime Calvo Consultant for: Bristol-Myers Squibb, Janssen, Celgene, Sanofi Genzyme, Speakers bureau: Bristol-Myers Squibb

DOI: 10.1136/annrheumdis-2019-eular.6053

\section{AB1227 PREFERRED REFERRAL PROTOCOL FOR RECENT ONSET ARTHRITIS IN ADULTS FROM PRIMARY CARE TO RHEUMATOLOGY}

Consuelo Ramos Giráldez ${ }^{1}$, María Espinosa ${ }^{2}$, Carolina Merino Argumánez ${ }^{3}$, Patricia Fernández Crespo ${ }^{4}$, Olga Rusinovich ${ }^{3}$, Fernando León Vázquez ${ }^{4}$, José Luis Andreu Sánchez ${ }^{3}$, Carmen Barbadillo Mateos ${ }^{3} .{ }^{1}$ Valme Hospital, Rheumatology Unit, Seville, Spain; ${ }^{2}$ Infanta Sofia Hospital, Rheumatology Unit, Madrid, Spain; ${ }^{3}$ Puerta de Hierro Majadahonda Hospital, Rheumatology Unit, Madrid, Spain; ${ }^{4}$ San Juan De la Cruz Primary Care Centre, Pozuelo de Alarcón (Madrid), Spain

Background: The time of rheumatoid arthritis (RA) evolution until treatment begins is key to controlling the disease. Many studies have shown that a prolonged duration of symptoms at the onset of treatment is associated with a more severe course of RA. The time from symptoms onset to first DMARD prescription is $>12$ weeks in Spain, because of diagnostic delay due to either patient-related factors (delay in consultation), Primary Care Physician (PCP) (delay in citation/referral) or rheumatologist (delay in citation).

Objectives: To evaluate the usefulness of teleconsulting as a preferred referral tool from PCP to Rheumatology for early detection, diagnosis and treatment of inflammatory joint disease in adults, in the health area of a tertiary hospital.

Methods: A preferential referral circuit was established between the PCP and the Rheumatology Service of a tertiary hospital, defining the referral criteria as "patient suspected of recent onset arthritis (ROA)": arthritis or inflammatory arthralgia in $>1$ peripheral joint for $>2$ weeks with neither traumatic cause nor previous diagnosis of rheumatic disease. PCP performed first assessment, the request for initial tests (blood test including acute phase reactants, rheumatoid factor \pm ANA, elemental urine and hands radiography) as well as the referral to Rheumatology with "ROA suspicion" motive. These consultation requests were cited from the Rheumatology Service within $<15$ days of receipt.

Before the protocol was established, its functioning was communicated as a face-to-face clinical session: by 2 rheumatologists in a Health Centre (HC) and by 1 PCP in the rest of the HC in the area. A poster was 
edited and published, exposing criteria and referral method for PCP's offices.

Results: During the first 6 months 33 patients were correctly referred. $78.8 \%$ were women and the average age was 49 years old. $48.8 \%$ (16 patients) were diagnosed with some inflammatory arthropathy: $31.5 \%$ were RA (5 cases); other diagnoses were arthropathy due to microcrystals deposit (2), overlap SLE/RA (1), MCTD(1), psoriatic arthritis (1), spondyloarthritis associated with IBD (1) or inflammatory arthralgia associated with retroperitoneal fibrosis (1). $12 \%$ (4 patients) were diagnosed with hands incipient osteoarthritis and $33.8 \%$ (12 patients) with arthralgias without data on inflammatory pathology. One patient missed her follow-up. The median time from symptom onset to Rheumatology assessment was 90 days in all patients; in the inflammatory pathologies subgroup, $68.8 \%$ was assessed in $<12$ weeks, with a median of 60 days and a mean of 96 days, starting treatment in this subgroup in the first assessment by Rheumatology.

Conclusion: In our experience, the establishment of a specific protocol in collaboration with Primary Care for the preferential referral of patients with suspected recent onset arthritis, obtained diagnoses not only for patients with rheumatoid arthritis, but also for other systemic autoimmune diseases in early stages, as well as early treatment initiation $(<12$ weeks) in most of these patients. In addition, it turned out to be an effective and comfortable referral route for PCP with suspected connective and/or inflammatory arthritis.

Disclosure of Interests: Consuelo Ramos Giráldez Speakers bureau: Sanofi, María Espinosa: None declared, Carolina Merino Argumánez: None declared, Patricia Fernández Crespo: None declared, Olga Rusinovich: None declared, Fernando León Vázquez: None declared, José Luis Andreu Sánchez: None declared, Carmen Barbadillo Mateos: None declared

DOI: 10.1136/annrheumdis-2019-eular.6144

\section{AB1228 ACCESS TO RHEUMATOLOGIC TREATMENTS AND AUTOIMMUNE CONDITIONS IN LATIN AMERICA}

Genessis Maldonado ${ }^{1}$, Daniella Cobeña ${ }^{1}$, Enrique Soriano ${ }^{2}$, Letty Moreno ${ }^{3}$, Carlos Rios ${ }^{1,3} .{ }^{1}$ Espiritu Santo University, Rheumatology, Guayaquil, Ecuador, ${ }^{2}$ Hospital Italiano de Buenos Aires, Buenos Aires, Argentina; ${ }^{3}$ Centro de Reumatología y Rehabilitación, Guayaquil, Ecuador

\section{Background:}

Objectives: To know the availability of treatment for rheumatologic and autoimmune conditions in Latin America, according to rheumatologists in the region.

Methods: A digital survey was created using Google Forms, it was approved and endorsed by the scientific committee of PANLAR, and later sent to the different Rheumatology associations of the region. The data was analyzed in the statistical program SPSS v.23.

Results: 456 surveys were filled by rheumatologists from 23 countries. The majority were females (54\%). The mean age was $47.18 \pm 11.79$ [25-78] years. $91 \%$ reported access to DMARDs, methotrexate 95\%, azathioprine $94 \%$, cyclophosphamide 92\%, hydroxychloroquine 92\%, leflunomide $90 \%$, mycophenolate mofetil $90 \%$, sulfazalasin $88 \%$, cyclosporine $80 \%$, tacrolimus $64 \%$, chloroquine $60 \%$. $85 \%$ have access to biological therapy: adalimumab $(78 \%)$, etarnercept $(83 \%)$, rituximab $(81 \%)$, infliximab $(74 \%)$, tocilizumab $(73 \%)$, abatacept $(67 \%)$, golimumab $(63 \%)$, certolizumab $(59 \%)$, secukinumab (55\%), ustekinumab (44\%), certolizumab (59\%), belimumab (47\%), canakinumab (22\%), sarilumab (15\%), ixekizumab $(12 \%)$, anakinra $(10 \%)$ and rilonacept $(3 \%)$. 58\% have access to biosimilar drugs: rituximab (27\%), infliximab $(24 \%)$, etanercept $(18 \%)$ and adalimumab (4\%). $61 \%$ have access to small molecules such as: tofacitinib $62 \%$, baricitinib $18 \%$ and ruxolutinib $1 \%$. Regarding the survey by each country 95\% reported access to DMARDs: between $80-100 \%$ (Argentina, Bolivia, Brazil, Chile, Colombia, Costa Rica, Cuba, Ecuador, El Salvador, Guatemala, Honduras, Mexico, Nicaragua, Panama, Paraguay, Peru, Puerto Rico, Dominican Republic). $70 \%$ of the countries reported access to biological therapy between $80-100 \%$ (Argentina, Bolivia, Brazil, Chile, Colombia, Costa Rica, Ecuador, El Salvador, Guatemala, Honduras, Panama, Paraguay, Puerto Rico, Uruguay.) $15 \%$ did not have access to biosimilar therapy (Cuba, Honduras and Uruguay). $30 \%$ have access to therapy with small molecules between $80-100 \%$ (Argentina, Guatemala, Honduras, Panama, Paraguay and Dominican Republic), 25\% did not have access to small molecules (Cuba, Uruguay and Venezuela).
Conclusion: It is evident that most Latin American countries have access to drugs focused on rheumatic diseases, however, there are substantial differences in countries that do not have access to all therapies. In addition, newer treatment as small molecules are less accessible to the region.

Disclosure of Interests: None declared

DOI: 10.1136/annrheumdis-2019-eular.7861

\section{AB1229 RHEUMATIC DISEASES REMISSION DURING PREGNANCY: MYTH OR REALITY? FACT OR HOPE?}

Euthalia Roussou ${ }^{1,2}$, Josie O'heney ${ }^{3}$, Victoria Sampson ${ }^{3}$, Asma Aziz $^{3} .{ }^{1}$ London, Rheumatology, Romford London, United Kingdom; ${ }^{2}$ Barking Havering and Redbridge University Hospitals NHS Trust, Rheumatology, Romford London, United Kingdom; ${ }^{3}$ Barking Havering and Redbridge University Hospitals NHS Trust, Obstetrics and Gynaecology, Romford, United Kingdom

Background: A Rheumatology Obstetric Combined Clinic (ROCC) has been running in our trust since 2013 for patients with any musculoskeletal (Msk) rheumatological disease. Observing pregnant ladies in this clinic it became noticeable that patients with msk diseases do not always go into remission during pregnancy.

Objectives: To register their perception of disease activity during pregnancy and from their memory of that disease activity before pregnancy A secondary aim was to assess whether the remission (or flare) during pregnancy was disease specific or trimester specific.

Methods: Patients with Msk disorders attending ROCC were given 2 different $10 \mathrm{~cm}$ visual analogue scales (VAS) asking them to grade the current (at the time of the assessment in ROCC) disease activity and their perceived disease activity prior to pregnancy. The recorded diseases were: (1) Lupus and other Connective Tissue Diseases $=18[(33.3 \%)$ of whom 13 were having Lupus and 5 Sjogren's syndrome, (2)SPAs =16 $[(28.6 \%)$ of whom 4 had Ankylosing Spondylitis (AS) and 12 Psoriatic arthritis (PsA)], (3) Rheumatoid Arthritis (RA) $=9$ (16.7), Antiphospholipid Syndrome $($ APS $)=7(13 \%)$ and other $=4(7.4 \%)$. In the "other" group there was 1 patients with sarcoidosis, 1 with Adult Onset Still's disease, 1 JIA which progressed to CREST, 1 knee pain. The last group was excluded from the analysis. SPSS was used for statistical analysis and chi square to assess the difference between the 2 groups

Results: We analyzed data from 50 patients who attended the ROCC. A the time of presentation mean age $( \pm s d)$ was 33.4 years $( \pm 4.29)$ range $22-43 y$ while age of disease presentation was $25.5( \pm 8.06)$. The weeks (w) of pregnancy during the time of assessment were (mean) $22.2( \pm 8.6)$ range 8-37 w. ESR (mean in $\mathrm{mmHg}$ ) was 27 ( \pm 21.7 ), CRP 13.6 ( \pm 14.18 ). Disease activity at presentation on a VAS was 4.24 ( \pm 2.8$)$ while reported disease activity prior to pregnancy was exactly the same of 4.24 ( \pm 3.07$)$.

Looking at groups of diseases RA and SpA patients showed reduction in the reported disease activity during pregnancy while CTDs and APS showed an increase, both of which however was not statistically significant (ss (table1)

\begin{tabular}{lccc}
\hline $\begin{array}{l}\text { Diseases (nr of } \\
\text { patients) }\end{array}$ & $\begin{array}{c}\text { Disease activity during } \\
\text { pregnancy } \\
\text { [mean }( \pm \text { sd)] }\end{array}$ & $\begin{array}{c}\text { Disease activity before } \\
\text { pregnancy } \\
\text { [mean }(+ \text { sd)] }\end{array}$ & $\begin{array}{c}\text { Statistical } \\
\text { significance }\end{array}$ \\
\hline CTDs (18) & $4.5(2.6)$ & $3.93(2.4$ & 0.6 \\
SpAs (16) & $4.6(2.8)$ & $5.8(3.9)$ & 0.8 \\
RA (9) & $4.13(2.5)$ & $4.75(2.5)$ & 0.9 \\
APS $(7)$ & $2.2(3.1)$ & $1(1.7)$ & 0.4 \\
\hline
\end{tabular}

Looking at DA according to trimester, it was in the 1st trimester (up to week 13) that patients were reported remision. (table 2 and figure 1).

\begin{tabular}{|c|c|c|c|c|c|}
\hline $\begin{array}{l}\text { Trimester } \\
\text { ( } \mathrm{n}=\text { number of } \\
\text { patients) }\end{array}$ & ESR & CRP & $\begin{array}{l}\text { Disease activity } \\
\quad \text { (current) }\end{array}$ & $\begin{array}{l}\text { Disease activity } \\
\quad \text { (before) }\end{array}$ & $\begin{array}{l}\text { Ss (chi } \\
\text { square) }\end{array}$ \\
\hline $1^{\text {st }}(\mathrm{n}=14)$ & $6(6.9)$ & $20(30)$ & 1.64 ( 2.09) & $2.5(2.7)$ & 0.02 \\
\hline $2^{\text {nd }}(n=19)$ & $\begin{array}{c}30.2 \\
(35.5)\end{array}$ & $\begin{array}{c}16.8(\mathrm{SD} \\
+13.5)\end{array}$ & $4.4(3.6)$ & $4.2(3.5)$ & 0.2 \\
\hline $3^{r d}(n=18)$ & $\begin{array}{c}17.3 \\
(20.3)\end{array}$ & $\begin{array}{c}3.9(\mathrm{sd} \\
+5.5)\end{array}$ & 3.67 (2.56) & $3.4(3.14)$ & 0.4 \\
\hline
\end{tabular}

